\title{
28 Research Square \\ PSMA-Targeting Redox Hyperbranched Poly (Amido Amine)-Mediated miR-133a-3p Delivery to Inhibit Bone Metastasis of Prostate Cancer
}

\section{Yongheng Ye}

Sun Yat-Sen University First Affiliated

\section{Lingli Zhang}

Depatrtment of Biomedical Engineering,Jinan University

\section{Yuhu Dai}

Sun Yat-sen University First Affiliated Hospital

\section{Zhi Wang}

Guangzhou institute of biomedicine and health,Chinese academy of sciences

\section{Cuie Li}

Guangzhou institute of biomedicine and health,Chinese academy of sciences

\section{Yue Peng}

Zhuhai City People's Hospital

\section{Dong Ma}

Department of Biomedical Engineering,Jinan University

\section{Peiheng He ( $\square$ hepeiheng@mail.sysu.edu.cn )}

Sun Yat-Sen University First Affiliated Hospital

\section{Research}

Keywords: miRNA, aptamer, hyperbranched polyamide amine, bone metastasis, prostate cancer

Posted Date: June 26th, 2020

DOI: https://doi.org/10.21203/rs.3.rs-36954/v1

License: (1) (1) This work is licensed under a Creative Commons Attribution 4.0 International License. Read Full License 


\section{Abstract}

Treatment of bone metastasis of prostate cancer remains a formidable challenge. The skeleton has a poorer blood supply, leading to inadequate drug distribution into the bone after administration. This study aimed to develop aptamer-anchored hyperbranched poly (amido amine) (HPAA) for the systemic delivery of miRNA-133a-3p and to evaluate its therapeutic potential against bone metastasis of prostate cancer in vivo and in vitro. A glutathione (GSH)-responsive cationic HPAA was prepared by the Michael addition reaction. Furthermore, HPAA-PEG was produced by PEGylation, and then the aptamer targeted to prostatespecific membrane antigen (PSMA) was conjugated to the HPAA-PEG. The obtained HPAA-PEG-APT could form nanocomplexes with miRNA-133a-3p through electrostatic adsorption. The results of immunocytochemistry indicated that the complexes could target PSMA-expressing LNCaP cells. The ability of HPAA-PEG-APT to facilitate the delivery of miRNA-133a-3p into LNCaP cells was proven, and HPAA-PEG-APT/miRNA-133a-3p demonstrated enhanced antitumor activity, lower cytotoxicity and better biocompatibility in vitro. Moreover, in a mouse tibial injection tumor model, the intravenous injection of the HPAA-PEG-APT/miRNA-133a-3p complex significantly inhibited cancer growth and extended the survival time. In summary, this study provided an aptamer-anchored HPAA-loaded gene system to deliver miRNA-133a-3p for better therapeutic efficacy of bone metastasis of prostate cancer.

\section{Introduction}

Prostate cancer $(\mathrm{PCa})$ is a high-incidence tumor of the male reproductive system ${ }^{[1]}$. Early-stage prostate cancer usually has a good prognosis, with a high 5-year survival rate ${ }^{2}$. However, advanced prostate cancer frequently metastasizes after chemotherapy, surgery and radiotherapy ${ }^{3}$. Bone is the most common distant metastatic site in PCa, mainly due to slow blood flow through the bone marrow and abundant adhesion receptors, growth factors and cytokines ${ }^{4-6}$. Serious complications accompany bone metastasis, such as pathological fracture, hypercalcemia, nerve compression syndrome and intractable pain ${ }^{7}$. Treatment mainly includes systemic treatment with endocrine therapy and chemotherapy, as well as local treatment for bone metastasis, such as surgery, radiotherapy, and bisphosphonates, which usually lead to drug resistance and harmful side effects ${ }^{[2,3]}$. The emergence of gene therapy has brought a new direction for the treatment of cancer ${ }^{[4,5]}$. The scope of gene therapy has been extended from genes to various nucleic acids such as antisense oligonucleotides (ASOs), siRNA, miRNA and mRNA ${ }^{[6-10]}$. Many lines of evidence have demonstrated that miRNA-based therapies hold great prospects in the treatment of $\mathrm{PCa}^{[7,11]}$. In our previous study, miRNA-133a-3p was proven to be a tumor suppressor gene in prostate cancer and downregulates several cytokine receptors associated with PCa growth, including FGFR, EGFR, IGFR and MET, inactivating the PI3K/AKT signaling pathway[12].

For gene delivery to the target tissue avoiding nucleic acid enzymatic degradation and immune recognition, the delivery systems were designed with better pharmacokinetics and biodistribution[13, 14]. Nonviral vectors seem a better choice than viral vectors to avoid the pathogenicity and immunogenicity of viral vectors. Among them, hyperbranched poly(amido amine)s (HPAAs) have attracted attention 
because they have good endosomal escape ability and can release drugs rapidly ${ }^{[15]}$. In particular, the expression of GSH has been confirmed to be much higher in tumor cells than in normal cells. Therefore, many studies, including our previous work, have developed redox HPAAs containing disulfide bonds, which were anticipated to cause endocellular degradation because of the breakdown of disulfide bonds under high GSH conditions and rapid drug release ${ }^{[16-20]}$.

To load genes into specific cells more effectively, targeting gene nanocarriers have been studied. PSMA is expressed at higher levels in advanced PCa, as well as bone metastasis of the disease ${ }^{[21]}$. Hence, PSMA is likely a promising target for the specific delivery of nanocarriers to $\mathrm{PCa}$, and mounting clinical trials have been demonstrated its imaging diagnosis and targeted therapy abilities ${ }^{[22,23]}$. The A $10-3.2$ aptamer (APT) has only 39 nucleotides, its weight is significantly reduced and it has good binding affinity to PSMA. Thus, many research teams have focused on APT-targeting gene delivery and favorable effects were reported $^{[11,24-26]}$.

In this study, we synthesized a multifunctional miRNA delivery nanocarrier comprising (1) miRNA-133a-3p used in treatment, (2) HPAAs used as miRNA-133a-3p nanocarriers, and (3) aptamers for specific tumor cell targeting (Scheme 1). We examined the capability of HPAA-PEG-APT to deliver miRNA-133a-3p. Additionally, we assess the treatment effect of the HPAA-PEG-APT/miRNA-133a-3p in vitro and in vivo.

\section{Experimental Details}

\subsection{Preparation of HPAA derivative}

\subsubsection{Materials}

CBA was synthesized based on our previous studies[17, 18]. 1-(2-Aminoethyl) piperazine (AEPZ) and dicyclohexylcarbodiimide (DCC) were obtained from Aladdin Industrial Corporation (Shanghai, China). aMalemidyl-w-N-hydroxysuccinimidyl polyethyleneglycol (MAL-PEG

-NHS, MW:5000) was purchased from Xi'an ruixi Biological Technology Co. Ltd. (Xi'an, China). The branched polyethylenimines (bPEls) were provided by Sigma-Aldrich. The solvents were provided by Chemical Reagent Factory (Guangzhou, China). A pLenti lentivirus was utilized for transfection and the generation of the LNCaP cell line to construct PSMA-expressing metastatic tumors animal models (OBiO, Shanghai, China). miRNA-133a-3p, negative control-miRNA and the A10-3.2 anti-PSMA aptamer modified with infrared dye Cy 5 at the 5 'terminus were purchased from RiboBio (Guangzhou, China).

\subsubsection{Preparation of HPAA-PEG and HPAA-PEG-APT}

HPAA was synthesized as described previously[17, 18]. First, $200 \mathrm{mg}$ of NHS-PEG-MAL was dissolved in phosphate-buffered solution (PBS, pH 7.4) for HPAA and NHS-PEG-MAL conjugation. Next, a 10:1 dilution of HPAA and NHS-PEG-MAL was conjugated through a specific reaction between the main amino groups of HPAA and NHS groups of the PEG derivative. The synthesis was performed in PBS at ambient 
temperature in the dark for $2 \mathrm{~h}$. Next, the HPAA-PEG conjugate was purified by ultrafiltration and then was freeze-dried and kept at $4{ }^{\circ} \mathrm{C}$ until ready for use.

To conjugate the anti-PSMA aptamer with HPAA-PEG, $100 \mathrm{mg}$ of HPAA-PEG was dissolved in PBS $(\mathrm{pH}$ 6.5-7.0). Next, $2 \mathrm{ml}$ of DMSO containing $1 \mathrm{mg}$ of APT was added dropwise to the HPAA-PEG and stirred in $30 \mathrm{~min}$, and the compound was reacted at ambient temperature in the dark for $48 \mathrm{~h}$. The HPAA-PEGAPT conjugate was also purified by ultrafiltration, followed by lyophilization and then storage at $4{ }^{\circ} \mathrm{C}$.

\subsection{Characterization}

\subsubsection{NMR}

The HPAA derivatives were characterized by nuclear magnetic resonance (NMR). Two milligrams each of HPAA, HPAA-PEG, NHS-PEG-MAL and HPAA-PEG-APT was dissolved in $\mathrm{D}_{2} \mathrm{O}$ and NMR. The results were compared using MestReNova software.

\subsubsection{Size and zeta potential}

The changes in the particle size and zeta potential of HPAA derivatives (with or without $10 \mathrm{mM} \mathrm{GSH}$ ) were characterized by dynamic light scattering (DLS; Zetasizer ZS90, Malvern). One milligram of samples was solubilized in high-purity water and tested at $25^{\circ} \mathrm{C}$. The morphology was visualized using a JEM2010HR transmission electron microscope.

\subsubsection{UV characterization}

The amount of APT in HPAA-PEG-APT was measured using UV-vis spectrophotometry, and the absorbance of APT was quantified at $260 \mathrm{~nm}$ using free APT as the reference standard.

\subsection{Gel electrophoresis}

To prepare HPAA-PEG-APT/miRNA complexes, the miRNA-133a-3p gene was mixed with HPAA-PEG-APT buffer at various HPAA-PEG-APT/miRNA mass ratios. For HPAA-PEG-APT, the gene-binding ability was tested by agarose gel electrophoresis. The result was visualized using a UV transilluminator (Bio-Rad, USA).

\subsection{Cell lines and culture}

The human PCa cell line LNCaP was provided by The Third Affiliated Hospital of Sun Yat-sen University. HUVECs were purchased from Shanghai Chinese Academy of Sciences cell bank (China). LNCaP and HUVECs were cultured as described previously[12].

\subsection{Specificity of the A $10-3.2$ aptamer}

The colocalization of Cy5 -labeled aptamer and anti-PSMA monoclonal antibodies (Abcam, Cambridge, MA, USA) in LNCaP cells was detected by LSCM.

\subsection{In vitro transfection}


LNCaP cells were cultured overnight before transfection in complete DMEM on 24-well plates $\left(5 \subseteq 10^{4}\right.$ cells/per well). Next, the cells were supplemented with Opti-MEM (without serum) containing HPAA or HPAA-PEG-APT complexes incubated with FAM-labeled miRNA (miRNA: $1 \mu \mathrm{g} /$ well) at different mass ratios $(60,80,100,120: 1)$. Thereafter, all the groups were supplemented with fresh DMEM for $6 \mathrm{~h}$, followed by culture for another 24-48 h. Next, the cells were washed with PBS and digested, following by centrifugation and resuspension using moderate PBS. The transfection efficiency was measured by flow cytometry (Accuri C6), and PEl-25 k/miRNA was used as the positive control.

\subsection{Anticancer effect in vitro}

The inhibitory effects of LNCaP cells were observed using the CCK-8 Kit (CCK-8; Beyotime, Shanghai, China). LNCaP cells were incubated in 96-microwell plates at $1 \times 10^{4}$ cells/well for $24 \mathrm{~h}$ at $37^{\circ} \mathrm{C}$. The cells were mixed with different concentrations $(0.02-50 \mu \mathrm{M})$ of complexes: HPAA-PEG-APT/miRNA, HPAA/miRNA and HPAA-PEG-APT/NCmiRNA for $72 \mathrm{~h}$. Subsequently, $10 \mu \mathrm{L}$ of CCK-8 was applied to all wells, followed by incubation for $4 \mathrm{~h}$. The absorbance of different wells was measured using a microplate reader (MULTISKAN MK3), a standard curve was plotted based on the absorbance and cell count, and each sample was measured three times. The $\mathrm{IC}_{50}$ value was counted using GraphPad 8.0 PRISM® ${ }^{[11]}$.

\subsection{Bcl2, EGFR and MET protein expression}

In total, $5 \subseteq 10^{4} \mathrm{LNCaP}$ cells were cultured in 6-well plates and incubated in $5 \% \mathrm{CO}_{2}$ at $37^{\circ} \mathrm{C}$ for $24 \mathrm{~h}$, reaching $80-90 \%$ confluence. Different samples (HPAA-PEG-APT/NCmiRNA, HPAA/miRNA and HPAAPEG-APT/miR-133a-3p, PBS) were mixed with the cells and reacted for $72 \mathrm{~h}$ (for protein extraction). Western blotting was performed using a previously described standard method ${ }^{24}$. Antibodies against Bcl2, EGFR and MET were purchased from Abcam (Cambridge, USA), and EGFR and MET were provided by Cell Signaling Technology.

\subsection{Cytotoxicity of HPAA derivatives for LNCaP cells}

The cytotoxicity of HPAA derivatives was observed using the CCK-8 assay in LNCaP and HUVEC lines. In total, $1 \times 10^{4}$ cells per well of 96 -well plates were seeded in DMEM. After $24 \mathrm{~h}$ of incubation, fresh media containing HPAA, HPAA-PEG and HPAA-PEG-APT at different concentrations (from 10 to $500 \mu \mathrm{g} / \mathrm{mL}$ ) were applied. Next, $100 \mu \mathrm{L}$ of fresh medium containing $10 \%$ CCK-8 was added to all wells, followed by washing with PBS. The absorbance was measured using a microplate reader at a $450-\mathrm{nm}$ wavelength, and the cell viability was defined as the ratio of the treated groups to the control group. Cell viability $(\%)=$ Absorbance of the treated group - Absorbance of the control group/Absorbance of the negative control group - Absorbance of the control group $\subseteq 100 \%$.

\subsection{Blood compatibility}

One-milliliter PBS solutions containing HPAA, HPAA-PEG, HPAA-PEG-APT, or PEl at different concentrations $(0.01 \mathrm{mg} / \mathrm{mL}, 0.5 \mathrm{mg} / \mathrm{mL}, 1 \mathrm{mg} / \mathrm{mL})$ were prepared, while pure water and the PBS group were used as the positive control and negative control, respectively. Next, $16 \%$ hematocrit (v/v) red blood 
cells (RBCs) suspended in PBS were mixed with $5 \mathrm{~mL}$ of PBS containing different concentrations of HPAA derivatives. The positive controls ( $100 \%$ hemolysis caused by PBS containing $5 \mathrm{~mL}$ of $0.1 \% \mathrm{NaCO}_{3}$ solution) and negative controls ( $0 \%$ hemolysis, only PBS) were prepared. The samples were incubated at $37^{\circ} \mathrm{Cfor} 1 \mathrm{~h}, 3 \mathrm{~h}, 6 \mathrm{~h}$, and $12 \mathrm{~h}$, followed by centrifugation for $5 \mathrm{~min}$ at $1000 \subseteq \mathrm{g}$ and measurement of the absorbance of the supernatant at $540 \mathrm{~nm}$ using a microplate reader. Finally, the ratio of hemolysis was determined by calculating the optical density (OD): Hemolysis (\%)=[(OD of the treated sample $-O D$ of negative control) $\subseteq 100] / O D$ of positive control. Each sample was tested in triplicate.

\subsection{Intraosseous model of prostate cancer and treatments}

All animal procedures were approved by The Institutional Animal Care and Use Committee of Sun Yat-sen University (No. L102012016110D). The bone metastasis animal model was induced by injecting LNCaPluc cells expressing the luciferase gene into the tibia ${ }^{4}$. Briefly, $5 \subseteq 10^{5} \mathrm{LNCaP}$-luc cells were collected from $25-\mathrm{cm}$ cell culture dishes (Corning, USA) and were resuspended in $20 \mu \mathrm{L}$ of PBS. The cell suspension was administered into the lumen of the tibia using a $0.1-\mathrm{mL}$ insulin needle. Tumor induction and its progression were investigated by estimating the bioluminescence signal intensity $\left(\mathrm{p} / \mathrm{s} / \mathrm{cm}^{2} / \mathrm{sr}\right)$. After 5min intraperitoneal injection of luciferin (200 mg/kg; VivoGlo ${ }^{\mathrm{Tm}}$ Luciferin; Promega, Madison, WI), the mice were imaged using IVIS ${ }^{\circledR}$ Lumina II (PerkinElmer, Waltham, MA). Two weeks following LNCaP cell injection, the mice were separated into 3 groups randomly with 5 mice per group. Next, 0.2-mL mixtures containing PBS (as control), HPAA-PEG-APT/miRNA-133a-3p, and HPAA/miRNA-133a-3p (HPAA derivatives: miRNA $=67 \mu \mathrm{g}: 33 \mu \mathrm{g}$ ) were administered via tail vein twice every 4 weeks. Tumor growth was observed by bioluminescence signal intensity and was monitored. Additionally, hematoxylin and eosin (H\&E) staining of the major organs (heart, lung, spleen, liver, kidney and prostate) was performed.

The criteria for the euthanasia of mice were as follows: $20 \%$ weight loss; movement difficulty because of tumor burden; tumor ulceration; necrosis; or bleeding. If the mice were also euthanized for signs of poor health, such as dehydration, lethargy, and cachexia, these mice were considered dead in the survival data.

\subsection{Statistical analysis}

All the data were expressed as means \pm standard deviation. Analyses of the difference between groups were performed by one-way analysis of variance (ANOVA) using GraphPad Prism 8 ). $P<0.05$ was defined as the significance level, ${ }^{*}$ for $P<0.05,{ }^{*}$ for $P<0.01$. All statistical tests were two-tailed.

\section{Results And Discussion}

\subsection{Synthesis and characterization of HPAA derivatives}

In this study, a GSH-response HPAA was prepared according to our previous work to form polymeric materials with specific targeting effects and gene codelivery capability [17, 18], and then HPAA was bonded with NHS-PEG-MAL through an amino-specific reaction. Furthermore, the anti-PSMA aptamer 
(APT) was conjugated with HPAA-PEG. Thereafter, the HPAA-PEG-APT vector could deliver miRNA-133a$3 p$ via electrostatic interactions between HPAA and miRNA.

The structure of HPAA derivatives was described by ${ }^{1} \mathrm{H}$ NMR, and the results are shown in Fig. $1 \mathrm{~A}$. All characterized peaks correspond to its chemical structure, indicating that HPAA derivatives had been synthesized successfully. The peaks of HPAA segments and MAL were from 2.3 to 3.5 and from 5.9 to 6.2, respectively. When the HPAA-PEG was conjugated with APT, the peaks from 5.9 to 6.2 were weakened, proving that some APT replaced the MAL (Fig. 1B). These above results showed that HPAAPEG-APT was synthesized successfully.

The APT content in HPAA-PEG-APT was tested using UV spectrophotometry. Free APT and HPAA-APT demonstrated a similar characteristic absorption peak at $260 \mathrm{~nm}$, proving that APT was conjugated with HPAA (Fig. 1C). A typical transmission electron microscopy (TEM) image of HPAA is shown in Fig. 1D, presenting a uniform size distribution of spheres.

The concentration of GSH in tumor cells is significantly higher than that outside the cells[27]. The structure of HPAA is rich in disulfide bonds, so HPAA-PEG-APT/miRNA is likely to exhibit excellent degradation performance at high intracellular GSH concentrations. The redox-responsive ability of the HPAA-PEG-APT/miRNA nanoparticles was assessed by measuring the particle size and zeta potentials using Zataszier Nano-ZS with or without $10 \mathrm{mM}$ GSH. In Fig. 1E, the particle size of HPAA-PEG-APT incubated with $10 \mathrm{mM} \mathrm{GSH}$ for 30 min was smaller than that of HPAA because of the cleavage of the disulfide bond. The GSH-responsive ability of the nanoparticle was helpful to release the gene in the tumor microenvironment. After crossing-linking by AEPZ, the zeta potential of HPAA increased from 0.8 to $25.6 \mathrm{mV}$ because of the aggregation of positive charge. A significant decrease in zeta potential $(20.7 \mathrm{mV})$ was observed in HPAA-PEG, which may be caused by the negatively charged groups in NHS-PEG-MAL. After conjugation with APT, the zeta potential of HPAA-PEG-APT returned to a positive charge $(3.8 \mathrm{mV})$ (Fig. 1F). Nanomaterials with neutral potentials have more effective bone marrow localization than those with anionic or cationic potentials because nanomaterials with neutral potentials have reduced interaction with serum proteins, unlike anionic or cationic nanomaterials that are opsonized to the same extent $^{[28-30]}$.

Figure 1. Synthesis and characterization of HPAA derivatives $(A){ }^{1} \mathrm{H}$ NMR spectra of HPAA, HPAA-PEGMAL and NHS-PEG-MAL. (B) ${ }^{1} \mathrm{H}$ NMR spectrum of HPAA-PEG-MAL and HPAA-PEG-APT. (C) UV-Vis spectrum of APT, HPAA-APT and HPAA. (D) TEM image of HPAA complexes. (E) Particle sizes and (F) potentials of HPAA derivatives $(n=3)$ incubated with or without GSH.

\subsection{The specificity of aptamers}

Using immunocytochemistry, the chemically synthesized A $10-3.2$ aptamer demonstrated its capacity for binding to prostate cancer cells with positive PSMA expression. In LNCaP cells, the Cy5-labeled HPAAPEG-APT complex colocalized on the cell membrane of LNCaP cells (Fig. 2A-H). 


\subsection{In vitro transfection}

Because of its hyperbranching structure and biodegradation properties, HPAA has also been presented to be an efficient gene delivery carrier in our previous studies ${ }^{[17,18]}$. To evaluate the miRNA delivery capacity of HPAA-PEG-APT, the gel retardation assay was used to detect its gene condensation capacity. When the weight ratio reached 5:1 or more, the miRNA was completely blocked, indicating that it was completely compressed and proving that HPAA-PEG-APT showed good in vitro gene compression ability (Fig. 3A). The rate of gene transfection of HPAA derivatives/miRNA complexes in LNCaP cells was assessed using in vitro transfection assays. The HPAA complexes all showed strong miRNA gene transfection, with transfection rates up to $80 \%$ (Fig. 3B). For HPAA-PEG-APT, its higher transfection efficiency was due to the negative charge of APT reducing the overall positive charge of the HPAA complex and its ability to bind to LNCaP cell surface PSMA antigen targets. However, once the mass ratio exceeded 120:1, its significant cytotoxicity led to a reduced transfection efficiency; thus, we chose a mass ratio of $80: 1$ for subsequent in vitro experiments.

Furthermore, flow cytometry revealed the transfection efficiency of miRNA-FAM into LNCaP cells was $92.4 \% \pm 1.2 \%$ after coincubation with HPAA-PEG-APT (Fig. 3C). By contrast, the transfection efficiency of miRNA-FAM into LNCaP cells co-incubated with HPAA was $82.4 \% \pm 2.3 \%$ through flow cytometry (Fig. 3C).

Figure 3. The rate of gene transfection of HPAA derivatives/miRNA complexes in vitro (A) Gel electrophoresis assay of HPAA-PEG-APT/miRNA with different weight ratios. (B) Treated with HPAA derivatives/miRNA ( $w / w=60,80,100,120)(n=3)$. (C) Detection of transfection efficiency between HPAA and HPAA-APT using FACS.

\subsection{Efficacy of treatments in vitro}

Similar to most tumor diseases, the leading cause of mortality of prostate cancer is related to metastasisrelated recurrence. Additionally, growing evidence suggests that activation of the PI3K/AKT signaling pathway plays a key factor in tumor metastasis ${ }^{[31-33]}$. In our previous work, miR-133a-3p prevents PCa bone metastasis by specifically targeting diverse cytokine receptors, including EGFR, FGFR1, IGF1R and MET, resulting in inactivation of the PI3K/AKT signaling pathway ${ }^{[12]}$. LNCaP cells were processed with HPAA-PEG-APT/miRNA, HPAA/miRNA and HPAA-PEG-APT/NCmiRNA in various concentrations. The results implied that cell growth was suppressed in a dose-dependent manner (Fig. 4A). The $\mathrm{IC}_{50}$ values were $132.8 \mathrm{nM}, 621.1 \mathrm{nM}$, and $2611 \mathrm{nM}$, respectively. Thus, HPAA-PEG-APT/miRNA has an IC ${ }_{50}$ value approximately 4.68 times lower than that of HPAA/miRNA, indicating that HPAA-PEG-APT could deliver more miRNAs into LNCaP cells. We further revealed the mechanism by studying the expression level of Bcl-2, MET, and EGFR protein and found that HPAA-PEG-APT efficiently transfects miR-133a-3p into LNCaP cells, specifically inhibiting the expression of the protein according to previous work ${ }^{12}$ (Fig. 4B).

Figure 4 In vitro anticancer effects (A)LNCaP Cell viabilities treated with HPAA-PEG-APT/miRNA, HPAA/miRNA and HPAA-PEG-APT/NCmiRNA, respectively, $(n=3)(B)$ The quantitative analysis of BCL 2, MET, and EFGR protein expression. 


\subsection{Biocompatibility}

\subsubsection{Blood safety}

In vivo, HPAA-PEG-APT should have good blood compatibility to obtain better bioavailability.

Spectrophotometric tests of the release of hemoglobin in erythrocytes were performed after treatment with HPAA derivatives to assess the blood compatibility of HPAA derivatives. Figure 5(A-D) presents the hemolysis ratios of different HPAA derivatives concentrations, with PEl as the control. The results showed that HPAA derivatives had good hemocompatibility. After $12 \mathrm{~h}$ incubation and a concentration of HPAAPEG-APT up to $0.5 \mathrm{mg} / \mathrm{mL}$, the sample still demonstrated nonhemolytic effects related to hemolysis ratios lower than the permissible level of $5 \%$. However, PEI showed severe hemolysis when the concentration of PEI was $0.5 \mathrm{mg} / \mathrm{mL}$, resulting in more than $70 \%$ hemolysis.

\subsubsection{Toxicity}

When HPAA-PEG-APT was used to treat cancer disease, its cytotoxicity should be evaluated first to confirm safety. The evaluation of cytotoxicity was performed using the CCK-8 assay, and the outcome is displayed in Fig. 6 . When the concentration of HPAA was more than $0.5 \mathrm{mg} / \mathrm{mL}$, it produced cytotoxicity. By contrast, HPAA-PEG-APT showed little cytotoxicity (Fig. 5E). When HPAA-PEG-APT carried the gene into the HUVECs, no cytotoxicity was detected (Fig. 5F).

Figure 5.The blood safety and toxicity of HPAA derivatives. (A-D)The hemolysis effect of the different concentrations of HPAA derivatives and PEI. (E)CCK-8 results of different concentrations of HPAA derivatives to LNCaP cells. (F) CCK-8 results of different concentrations of HPAA derivatives to HUVEC cells.

\subsection{Efficacy of treatments in vivo}

The therapeutic effect of HPAA-PEG-APT/miRNA-133a-3p and HPAA/miRNA-133A-3p were explored in a mouse tibial injection tumor model. The representative tumor profile image and tumor growth after different drug treatments are shown in Fig. 6. HPAA-PEG-APT/miRNA-133a-3p and HPAA/miRNA-133A-3p demonstrated significant anti-tumor effects. Particularly, the tumors treated with HPAA-PEG-APT/miRNA133a-3p showed a substantially smaller tumor volume those treated with HPAA/miRNA-133A-3p (Fig. 6.A). The mice in the HPAA-PEG-APT/miRNA-133a-3p, HPAA/miRNA-133A-3p and control groups died on days 70,55 , and 42 , respectively. The anti-tumor effect of the HPAA-PEG-APT/miRNA group was significantly more effective than that of the other groups; $50 \%$ of the mice survived more than 83 days, with a maximum survival time of 88 days (Fig. 6B). The mice were injected with PBS, HPAA/miRNA-133a$3 p$ and HPAA-PEG-APT/miRNA-133a-3p twice a week for 4 weeks, and the bioluminescent signals of the HPAA/miRNA-133a-3p and HPAA-PEG-APT/miRNA-133a-3p groups were lower than those of the untreated group (Fig. 6C). Additionally, the anti-tumor effect of HPAA equipped with APT was more pronounced because of the targeting effect provided by APT, and the nanocomposite can deliver the therapeutic gene to the tumor microenvironment more efficiently in vivo. The in vivo toxicity of HPAA was assessed by H\&E staining of vital organs in mice, and no significant difference was found in organ or 
histology damage between the HPAA and saline groups (Fig. 6D), further demonstrating the safety of HPAA-PEG-APT. The above results showed that HPAA-PEG-APT/miRNA-133a-3p is more effective in inhibiting bone resorption.

Figure 6 In vivo treatment efficacy of HPAA complexes (A) Tumor growth curve in the different groups. (B) Cumulative survival outcome of in the various treatment groups. (C) Representative bioluminance signal of bone metastasis of a mice at various treatment groups after 8 weeks. (D) representative HE staining of organ histology at various treatment groups. Scale bars:100 $\mu \mathrm{m}$

\section{Conclusion}

We successfully constructed HPAA-PEG-APT, which demonstrated good affinity and specificity for PSMApositive prostate cancer cells and bone metastasis of prostate cancer in vitro and in vivo by loading miRNA-133a-3p. In particular, a self-sensibilized platform with a gene targeting strategy showed a highefficiency anti-tumor effect on bone metastasis of prostate cancer tumors and may be a promising gene therapy for cancers.

\section{Abbreviations}

PSMA

prostate-specific membrane antigen;HPAA:hyperbranched poly (amido amine); miRNA:MicroRNA; GSH:glutathione; APT:aptamer; PCa:Prostate cancer; ASOs:antisense oligonucleotides; Bcl2:B-cell CLL/lymphoma 2;FGFR:Fibroblast Growth Factor Receptor,;EGFR:Epidermal Growth Factor Receptor; IGFR:Insulin Like Growth Factor Receptor; MET:MET Proto-Oncogene, Receptor Tyrosine Kinase; CBA:N,NO-cystaminebisacrylamide; AEPZ:1-(2-Aminoethyl) piperazine ; DCC:dicyclohexylcarbodiimide;MAL-PEG-NHS:Malemidyl-w-N-hydroxysuccinimidyl polyethyleneglycol ; HPAA-APT:APT-load HPAA;HPAA-PEG-APT/miRNA:APT- and miRNA-133a-3p-loaded HPAA; NMR:nuclear magnetic resonance; OD:optical density; H\&E:hematoxylin and eosin;

\section{Declarations}

\section{Acknowledgements}

Not applicable

\section{Funding}

Not applicable

\section{Author's contributions}

Yongheng Ye wrote the manuscript. Lingli Zhang synthesized HPAA-PEG-APT and Yuhu Dai was in charge of in vivo experiments. Zhi Wang and Cuie Li conducted the animal experiments. Yue Peng 
contributed to the analysis of data. Dong Ma and Peiheng He developed ideas and drafted the manuscript.

\section{Availability of data and materials}

The datasets used and /or analyzed during the current study are available from the corresponding author on reasonable request.

\section{Ethics approval and consent to participate}

Not applicable

\section{Consent for publication}

Not applicable

\section{Competing interest}

The authors declare no conflict of interest

\section{Author details}

${ }^{1}$ Department of orthopedic surgery, Guangdong Prov Key Lab Orthopaed \& Traumatol, the First Affiliated Hospital of Sun Yat-sen University, Guangzhou, Guangdong Province, 510080, China. ${ }^{2}$ Key Laboratory of Biomaterials of Guangdong Higher Education Institutes, Department of Biomedical Engineering, Jinan University, Guangzhou, 510632, China.${ }^{3}$ Guangzhou institute of biomedicine and health, Chinese academy of sciences, Guangzhou, Guangdong Province, 510530, China. ${ }^{4}$ Department of Otorhinolaryngology Head and Neck Surgery, Zhuhai People's Hospital (Zhuhai hospital affiliated with Jinan University), Zhuhai, Guangdong Province, 519000, China

\section{References}

1. Siegel RL, Miller KD, Jemal A. Cancer statistics, 2018. CA Cancer J Clin. 2018;68:7-30.

2. Adjei IM, Temples MN, Brown SB, Sharma B. Targeted Nanomedicine to Treat Bone Metastasis. Pharmaceutics 2018, 10.

3. Saad F, Lipton A, Cook R, Chen YM, Smith M, Coleman R. Pathologic fractures correlate with reduced survival in patients with malignant bone disease. Cancer. 2007;110:1860-7.

4. Altwaijry N, Somani S, Dufès C. Targeted nonviral gene therapy in prostate cancer. Int J Nanomed. 2018;Volume 13:5753-67.

5. Mollaei H, Safaralizadeh R, Rostami Z. MicroRNA replacement therapy in cancer. J Cell Physiol. 2019;234:12369-84. 
6. Chen J, Wu Z, Ding W, Xiao C, Zhang Y, Gao S, Gao Y, Cai W. SREBP1 siRNA enhance the docetaxel effect based on a bone-cancer dual-targeting biomimetic nanosystem against bone metastatic castration-resistant prostate cancer. Theranostics. 2020;10:1619-32.

7. Ma D, Liu H, Zhao P, Ye L, Zou H, Zhao X, Dai H, Kong X, Liu P. Programing Assembling/Releasing Multifunctional miRNA Nanomedicine to Treat Prostate Cancer. ACS Appl Mater Interfaces. 2020;12:9032-40.

8. Zhang X, He Z, Xiang L, Li L, Zhang H, Lin F, Cao H. Codelivery of GRP78 siRNA and docetaxel via RGD-PEG-DSPE/DOPA/CaP nanoparticles for the treatment of castration-resistant prostate cancer. Drug Des Devel Ther. 2019;13:1357-72.

9. Wong JKL, Mohseni R, Hamidieh AA, MacLaren RE, Habib N, Seifalian AM: Will Nanotechnology Bring New Hope for Gene Delivery? Trends Biotechnol 2017, 35:434-451.

10. Gu J, Chen X, Ren X, Zhang X, Fang X, Sha X. CD44-Targeted Hyaluronic Acid-Coated RedoxResponsive Hyperbranched Poly(amido amine)/Plasmid DNA Ternary Nanoassemblies for Efficient Gene Delivery. Bioconjug Chem. 2016;27:1723-36.

11. Wu X, Ding B, Gao J, Wang H, Fan W, Wang X, Zhang W, Wang X, Ye L, Zhang M, et al. Secondgeneration aptamer-conjugated PSMA-targeted delivery system for prostate cancer therapy. Int J Nanomedicine. 2011;6:1747-56.

12. Tang Y, Pan J, Huang S, Peng X, Zou X, Luo Y, Ren D, Zhang X, Li R, He P, Wa Q. Downregulation of miR-133a-3p promotes prostate cancer bone metastasis via activating PI3K/AKT signaling. J Exp Clin Cancer Res. 2018;37:160.

13. Zhang Y, Wang Z, Gemeinhart RA. Progress in microRNA delivery. J Control Release. 2013;172:96274.

14. Blanco E, Shen H, Ferrari M. Principles of nanoparticle design for overcoming biological barriers to drug delivery. Nat Biotechnol. 2015;33:941-51.

15. Conte R, Valentino A, Di Cristo F, Peluso G, Cerruti P, Di Salle A, Calarco A. Cationic Polymer Nanoparticles-Mediated Delivery of miR-124 Impairs Tumorigenicity of Prostate Cancer Cells. Int J Mol Sci 2020, 21.

16. Liu T, Li J, Wu X, Zhang S, Lu Z, Li G, Li J, Chen S. Transferrin-targeting redox hyperbranched poly(amido amine)-functionalized graphene oxide for sensitized chemotherapy combined with gene therapy to nasopharyngeal carcinoma. Drug Deliv. 2019;26:744-55.

17. Tang Q, Ma X, Zhang Y, Cai X, Xue W, Ma D. Self-sensibilized polymeric prodrug co-delivering MMP-9 shRNA plasmid for combined treatment of tumors. Acta Biomater. 2018;69:277-89.

18. Li M, Zhou X, Zeng X, Wang C, Xu J, Ma D, Xue W. Folate-targeting redox hyperbranched poly(amido amine)s delivering MMP-9 siRNA for cancer therapy. Journal of Materials Chemistry B. 2016;4:54756.

19. Liu F, Lin L, Sheng S, Xu C, Wang Y, Zhang Y, Wang D, Wu J, Li Y, Tian H, Chen X. A glutathionedepleting chemodynamic therapy agent with photothermal and photoacoustic properties for tumor theranostics. Nanoscale. 2020;12:1349-55. 
20. Zhuang Y, Deng H, Su Y, He L, Wang R, Tong G, He D, Zhu X. Aptamer-Functionalized and Backbone Redox-Responsive Hyperbranched Polymer for Targeted Drug Delivery in Cancer Therapy. Biomacromol. 2016;17:2050-62.

21. Vogel MME, Kroeze SGC, Henkenberens C, Schmidt-Hegemann NS, Kirste S, Becker J, Burger IA, Derlin T, Bartenstein $\mathrm{P}, \mathrm{Mix} \mathrm{M}$, et al: Prognostic risk classification for biochemical relapse-free survival in patients with oligorecurrent prostate cancer after [(68)Ga]PSMA-PET-guided metastasis-directed therapy. Eur J Nucl Med Mol Imaging 2020.

22. Hofman MS, Lawrentschuk N, Francis RJ, Tang C, Vela I, Thomas P, Rutherford N, Martin JM, Frydenberg $M$, Shakher $\mathrm{R}$, et al: Prostate-specific membrane antigen PET-CT in patients with high-risk prostate cancer before curative-intent surgery or radiotherapy (proPSMA): a prospective, randomised, multi-centre study. Lancet 2020.

23. Derlin T, Werner RA, Lafos M, Henkenberens C, von Klot CAJ, Sommerlath Sohns J, Ross TL, Bengel FM. Neuroendocrine Differentiation and Response to PSMA-Targeted Radioligand Therapy in Advanced Metastatic Castration-Resistant Prostate Cancer: a Single-Center Retrospective Study. J Nucl Med 2020.

24. Xu W, Siddiqui IA, Nihal M, Pilla S, Rosenthal K, Mukhtar H, Gong S. Aptamer-conjugated and doxorubicin-loaded unimolecular micelles for targeted therapy of prostate cancer. Biomaterials. 2013;34:5244-53.

25. Fan X, Guo Y, Wang L, Xiong X, Zhu L, Fang K. Diagnosis of prostate cancer using anti-PSMA aptamer A10-3.2-oriented lipid nanobubbles. Int J Nanomedicine. 2016;11:3939-50.

26. Wu M, Zhao H, Guo L, Wang Y, Song J, Zhao X, Li C, Hao L, Wang D, Tang J. Ultrasound-mediated nanobubble destruction (UMND) facilitates the delivery of A10-3.2 aptamer targeted and siRNAloaded cationic nanobubbles for therapy of prostate cancer. Drug Deliv. 2018;25:226-40.

27. Xu X, Wu J, Liu S, Saw PE, Tao W, Li Y, Krygsman L, Yegnasubramanian S, De Marzo AM, Shi J, et al. Redox-Responsive Nanoparticle-Mediated Systemic RNAi for Effective Cancer Therapy. Small. 2018;14:e1802565.

28. Vijayaraghavalu S, Gao Y, Rahman MT, Rozic R, Sharifı N, Midura RJ, Labhasetwar V. Synergistic combination treatment to break cross talk between cancer cells and bone cells to inhibit progression of bone metastasis. Biomaterials. 2020;227:119558.

29. Adjei IM, Sharma B, Peetla C, Labhasetwar V. Inhibition of bone loss with surface-modulated, drugloaded nanoparticles in an intraosseous model of prostate cancer. J Control Release. 2016;232:8392.

30. Arvizo RR, Miranda OR, Moyano DF, Walden CA, Giri K, Bhattacharya R, Robertson JD, Rotello VM, Reid JM, Mukherjee P. Modulating pharmacokinetics, tumor uptake and biodistribution by engineered nanoparticles. PLoS One. 2011;6:e24374.

31. Zhang XH, Jin X, Malladi S, Zou Y, Wen YH, Brogi E, Smid M, Foekens JA, Massague J. Selection of bone metastasis seeds by mesenchymal signals in the primary tumor stroma. Cell. 2013;154:106073. 
32. Jiang WG, Sanders AJ, Katoh M, Ungefroren H, Gieseler F, Prince M, Thompson SK, Zollo M, Spano D, Dhawan P, et al. Tissue invasion and metastasis: Molecular, biological and clinical perspectives. Semin Cancer Biol. 2015;35(Suppl:):244-75.

33. Polivka J Jr, Janku F. Molecular targets for cancer therapy in the PI3K/AKT/mTOR pathway. Pharmacol Ther. 2014;142:164-75.

\section{Figures}

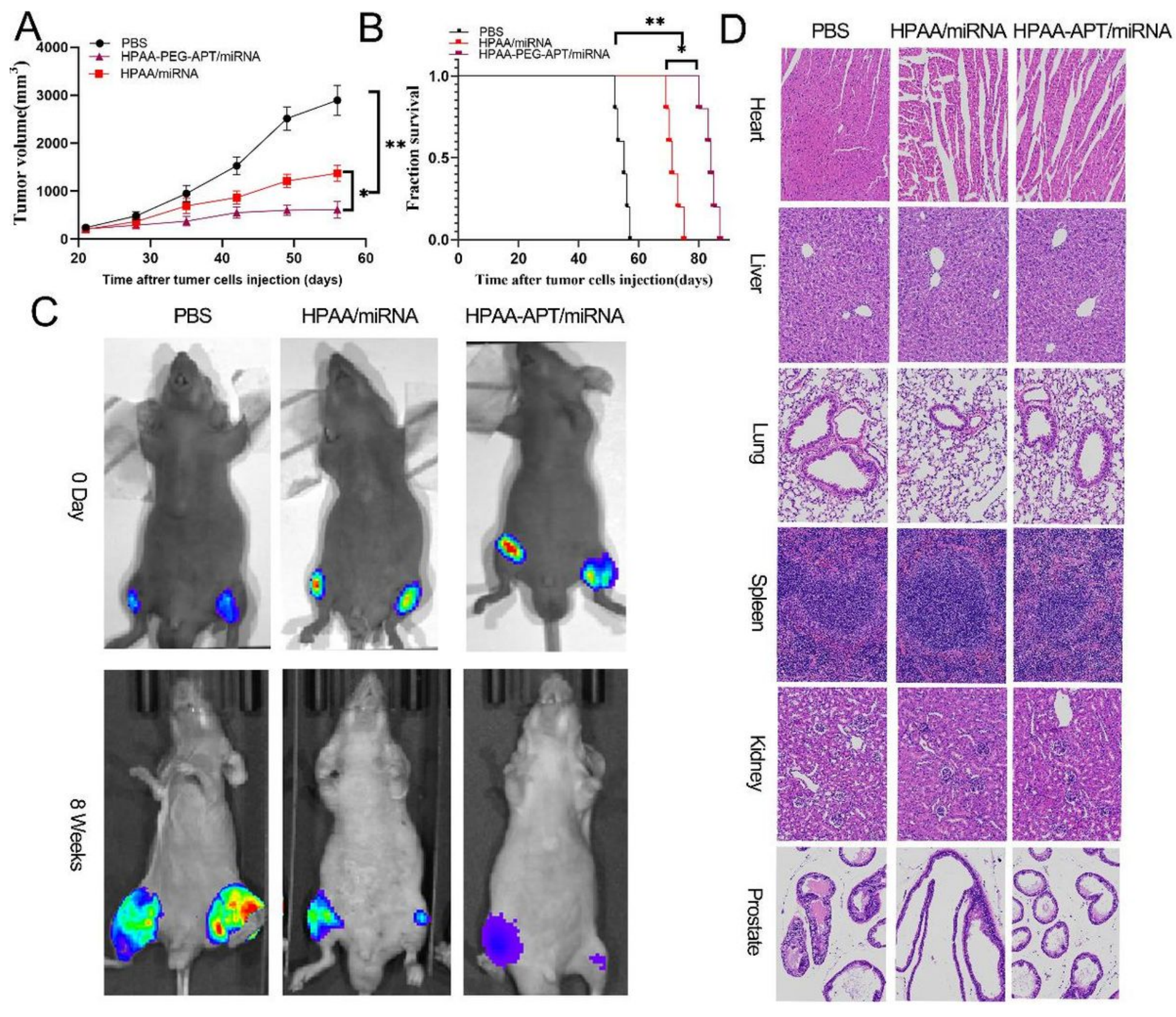

\section{Figure 1}

In vivo treatment efficacy of HPAA complexes (A) Tumor growth curve in the different groups. (B) Cumulative survival outcome of in the various treatment groups. (C) Representative bioluminance signal 
of bone metastasis of a mice at various treatment groups after 8 weeks. (D) representative HE staining of organ histology at various treatment groups. Scale bars:100 $\mu \mathrm{m}$

A

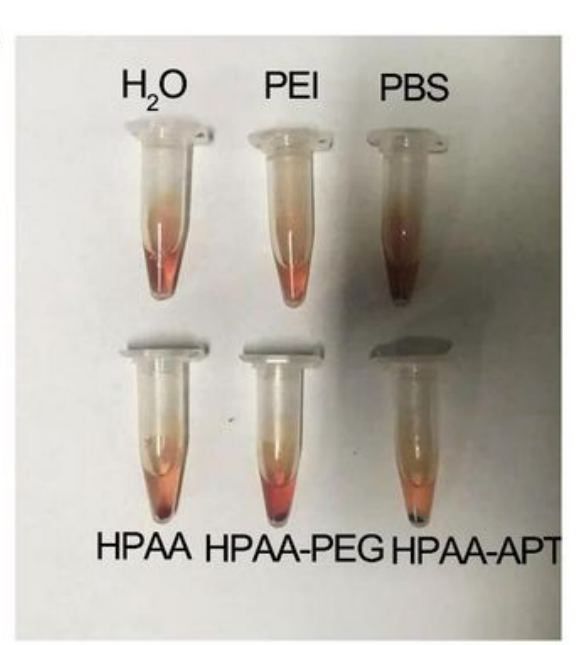

C
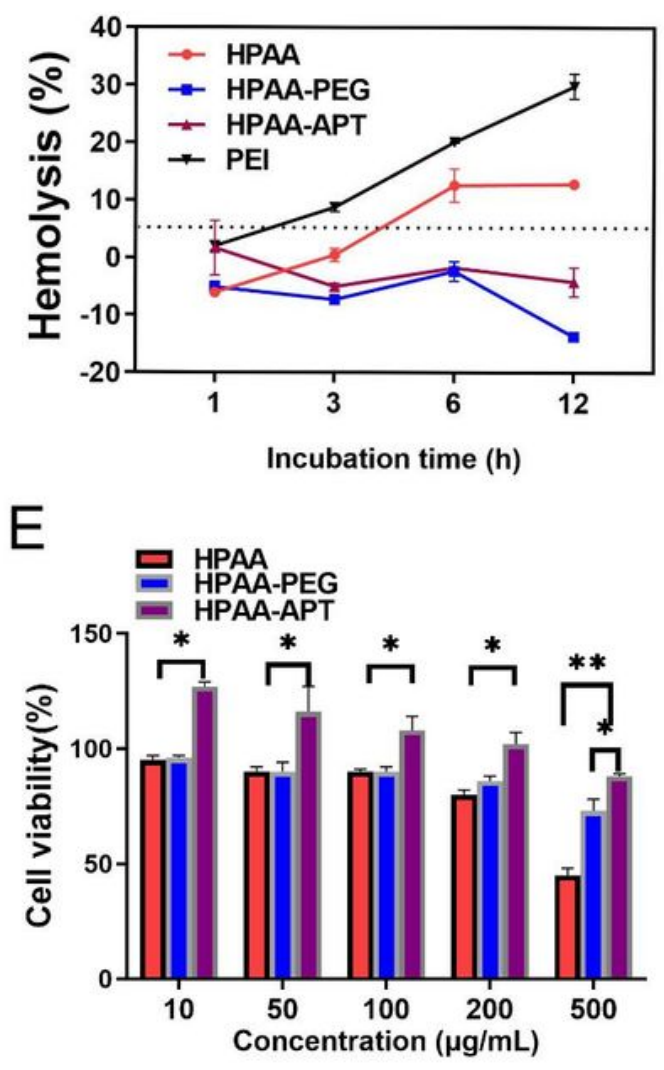

B

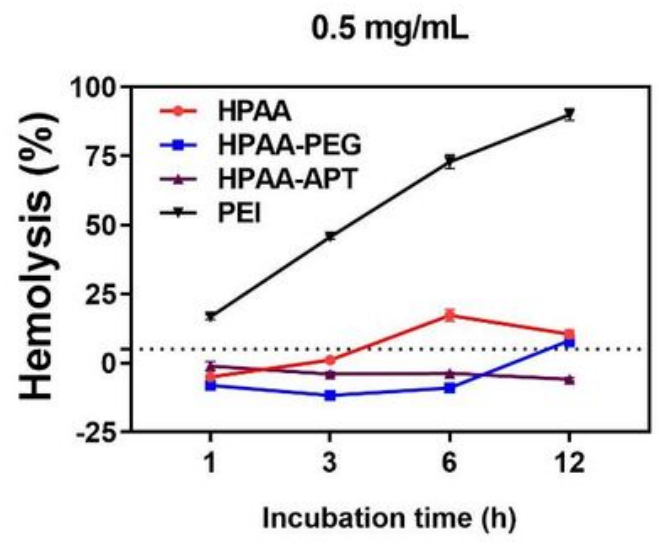

D

$0.01 \mathrm{mg} / \mathrm{mL}$

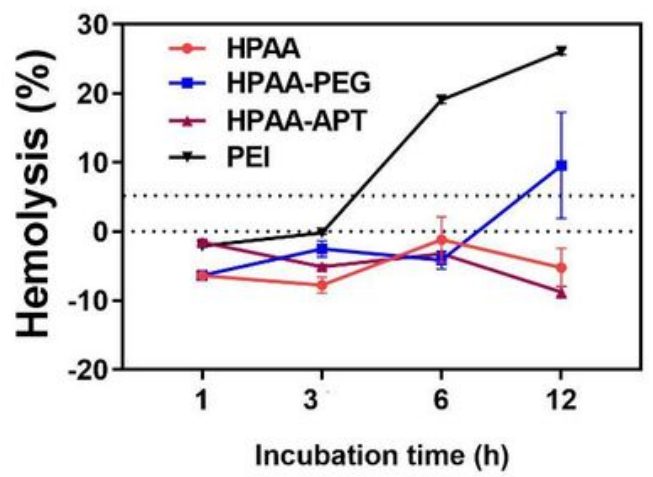

$\mathrm{F}$

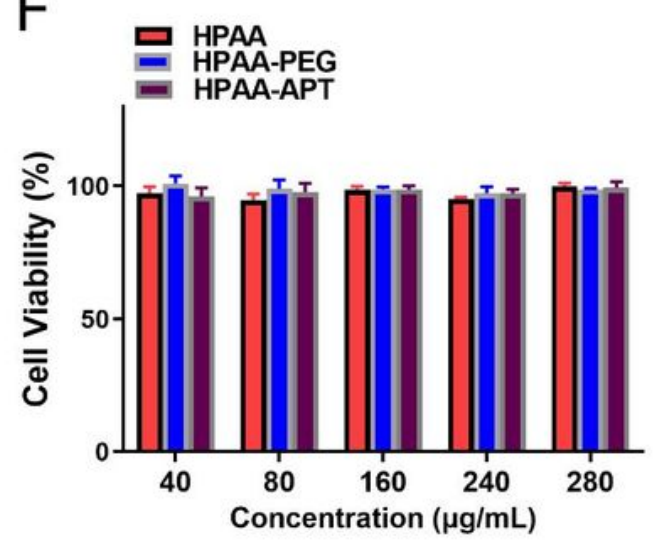

Figure 2

The blood safety and toxicity of HPAA derivatives . (A-D)The hemolysis effect of the different concentrations of HPAA derivatives and PEI. (E)CCK-8 results of different concentrations of HPAA 
derivatives to LNCaP cells. (F) CCK-8 results of different concentrations of HPAA derivatives to HUVEC cells.

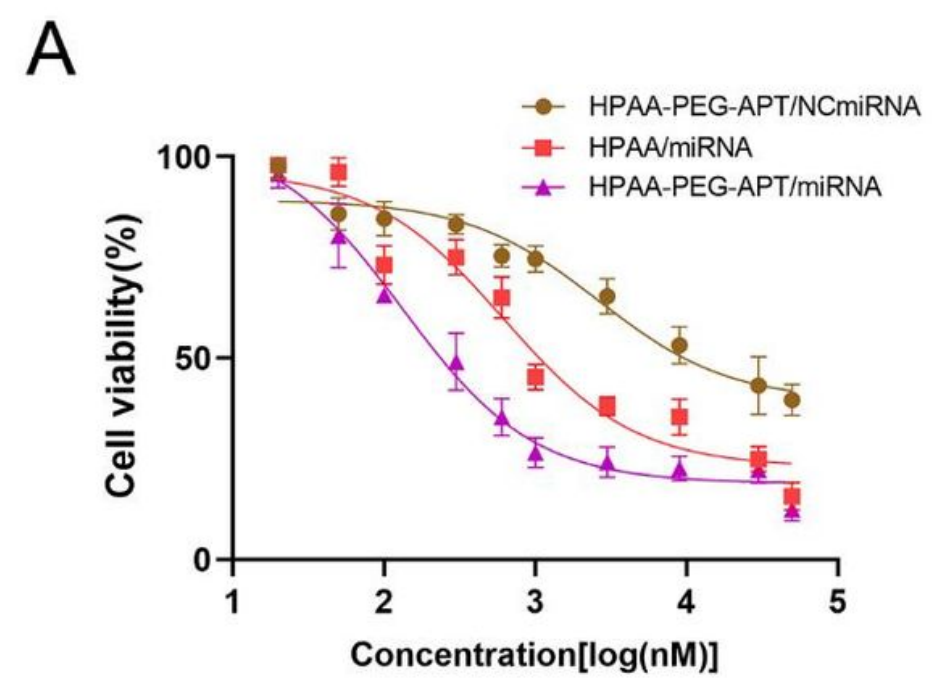

B

\section{Figure 3}

In vitro anticancer effects (A)LNCaP Cell viabilities treated with HPAA-PEG-APT/miRNA, HPAA/miRNA and HPAA-PEG-APT/NCmiRNA, respectively, $(n=3)(B)$ The quantitative analysis of BCL 2, MET, and EFGR protein expression. 


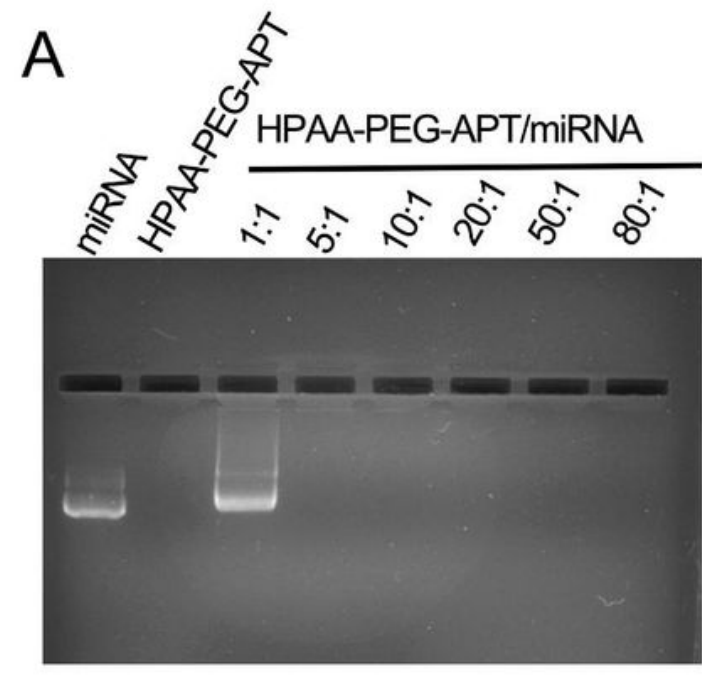

C

HPAA/miRNA 80:1

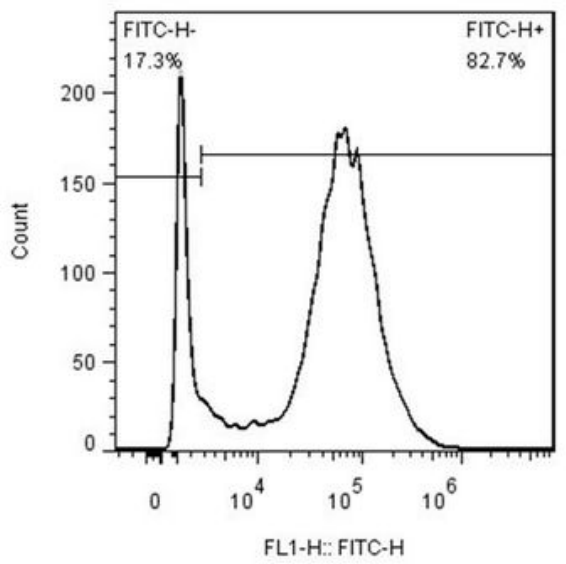

PEI/miRNA 80:1

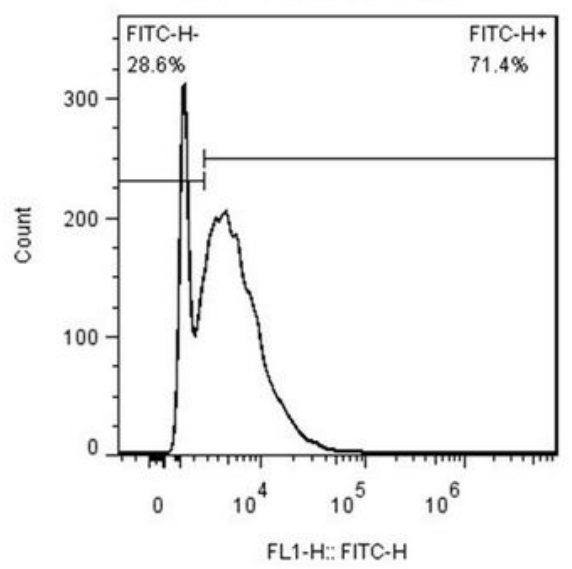

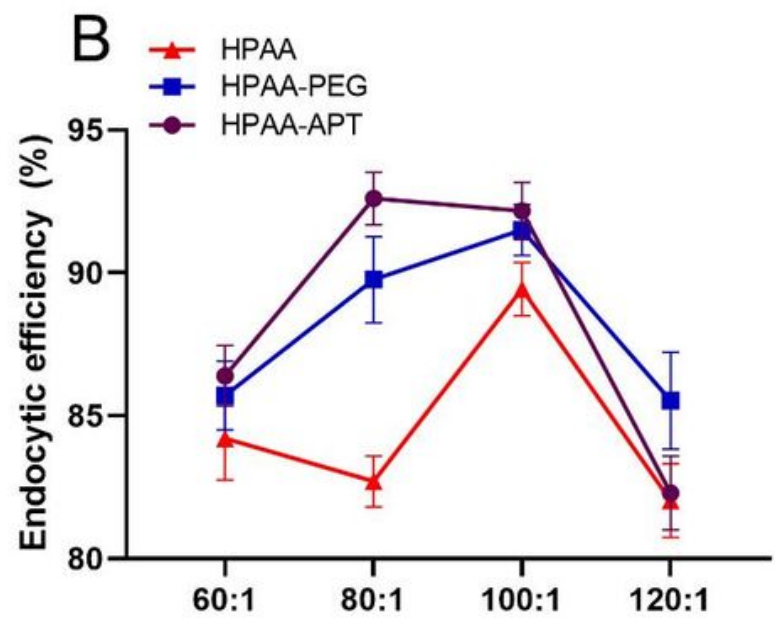

HPAA derivatives/miRNA $(\mathrm{w} / \mathrm{w}=60,80,100,120)$

HPAA-APT/miRNA 80:1
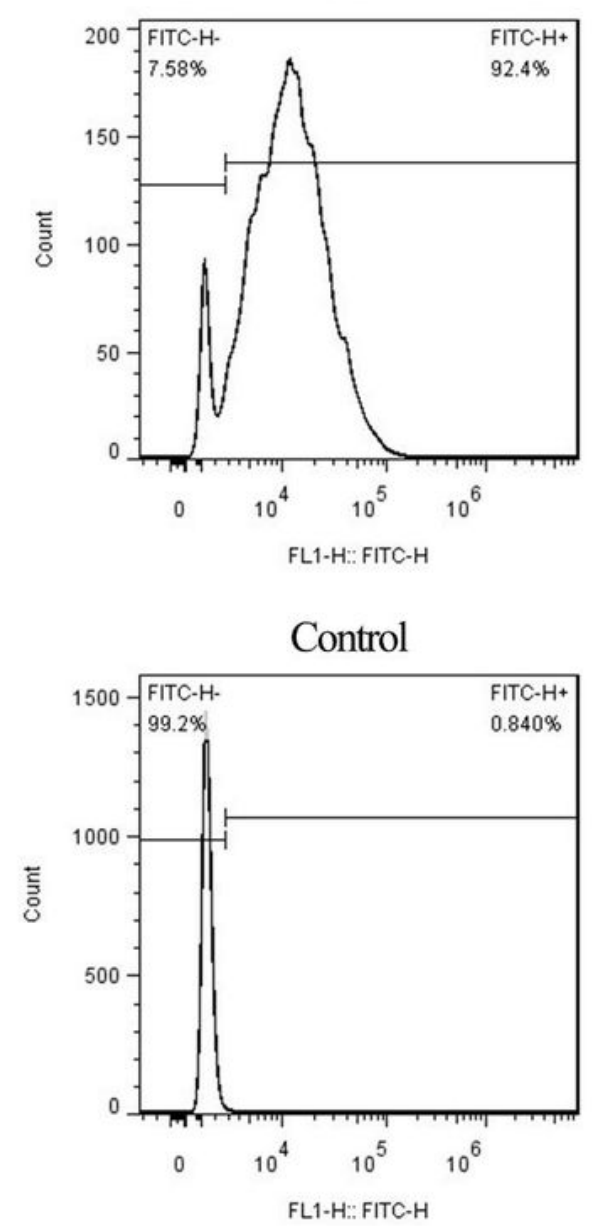

\section{Figure 4}

The rate of gene transfection of HPAA derivatives/miRNA complexes in vitro (A) Gel electrophoresis assay of HPAA-PEG-APT/miRNA with different weight ratios. (B) Treated with HPAA derivatives/miRNA $(w / w=60,80,100,120)(n=3) .(C)$ Detection of transfection efficiency between HPAA and HPAA-APT using FACS. 


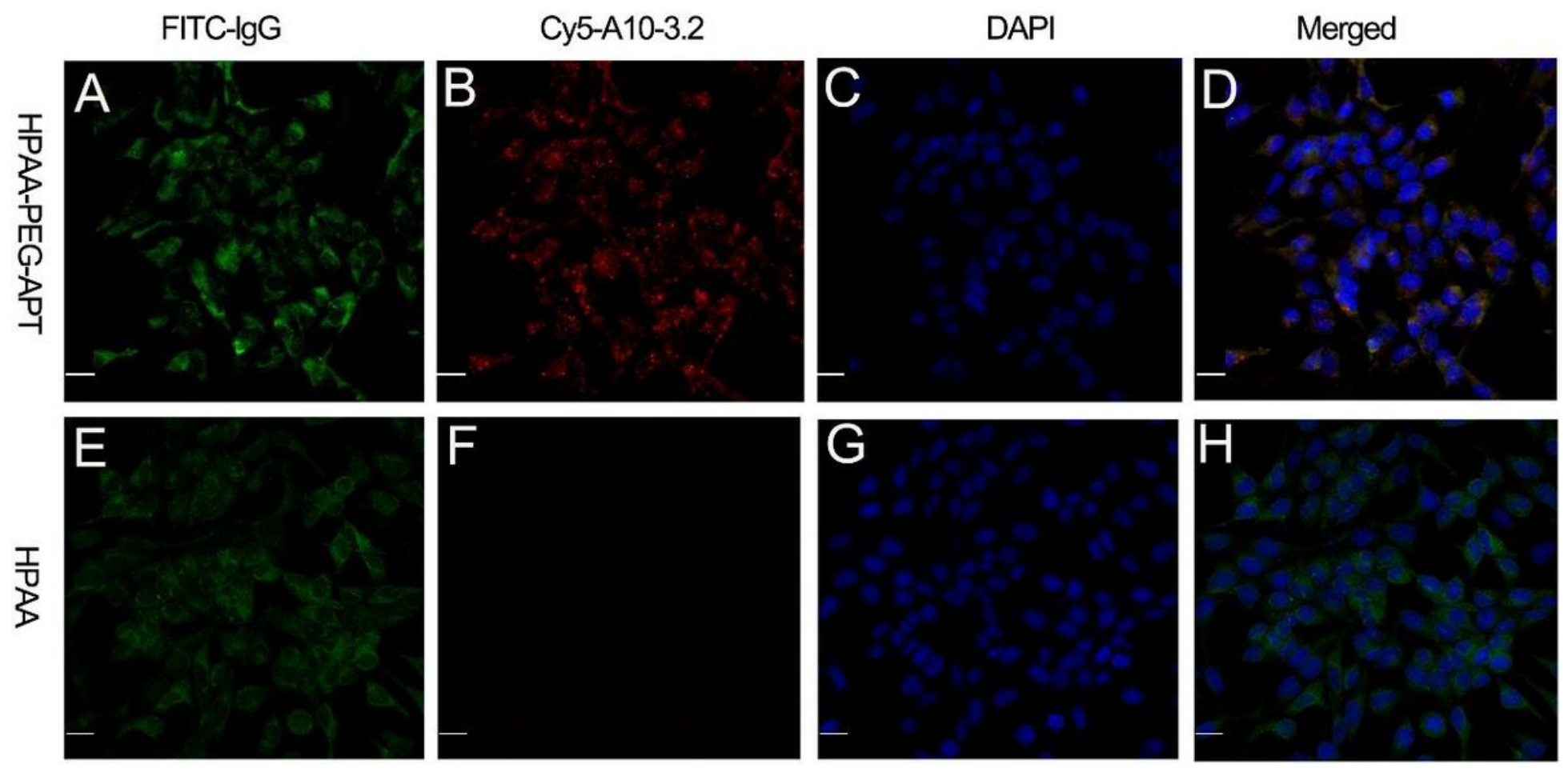

Figure 5

The specificity of the aptamer in LNCaP cells . (A, E) Identification of PSMA expression in LNCaP cells via indirect immunofluorescence uing anti-PSMA monoclonal antibody and FITC-labeled IgG secondary. (B, F) Identification of Cy5-labeled A 10-3.2 aptamer binding to LNCaP cells using direct immunochemistry. $(C, G)$ cell nuclei with DAPI-stained. (D, H) Merged fluorescence images. Scale bars:50 $\mu \mathrm{m}$ 

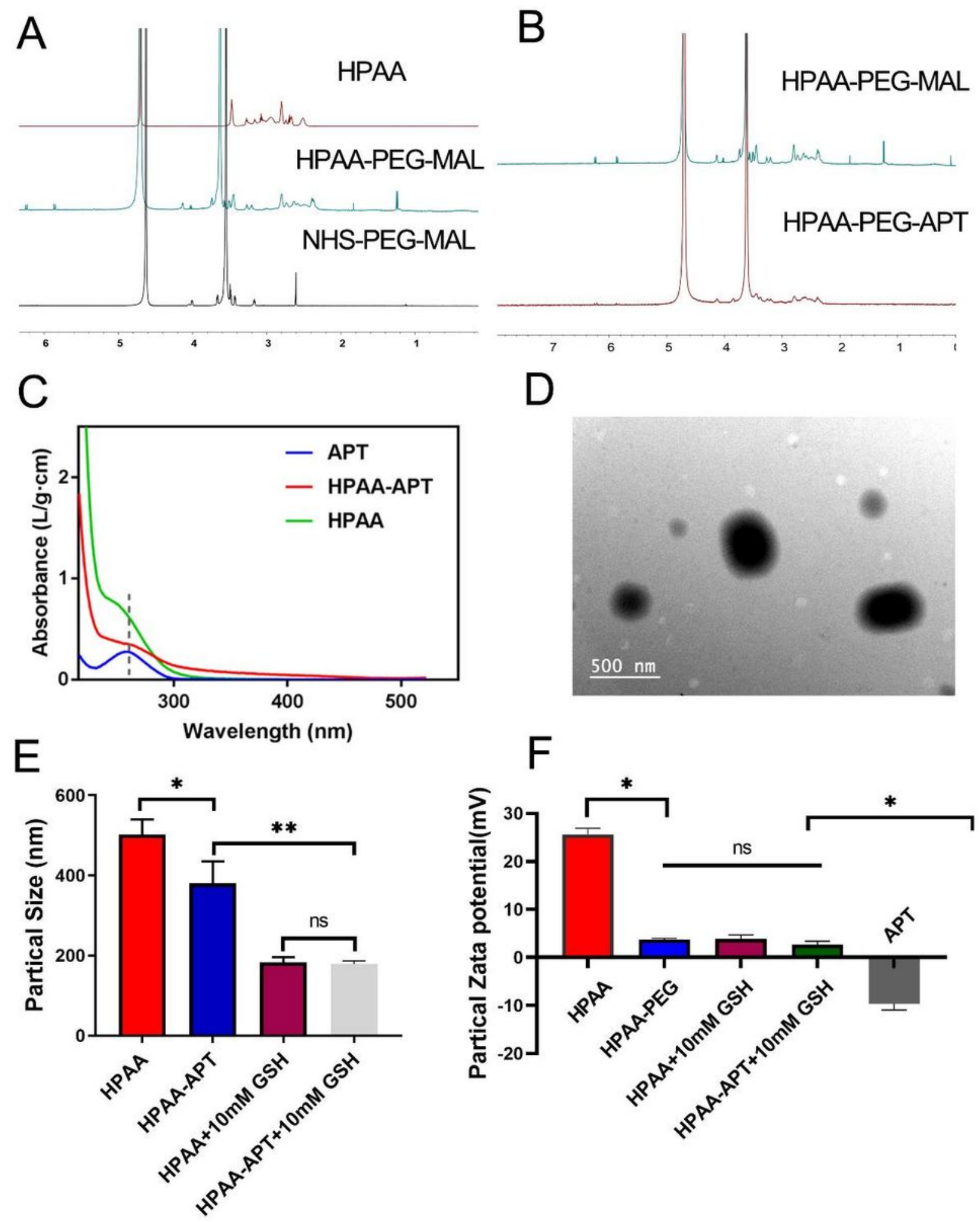

Figure 6

Synthesis and characterization of HPAA derivatives (A) 1H NMR spectra of HPAA, HPAA-PEG-MAL and NHS-PEG-MAL. (B) 1H NMR spectrum of HPAA-PEG-MAL and HPAA-PEG-APT. (C) UV-Vis spectrum of APT, HPAA-APT and HPAA. (D) TEM image of HPAA complexes. (E) Particle sizes and (F) potentials of HPAA derivatives $(n=3)$ incubated with or without $G S H$. 

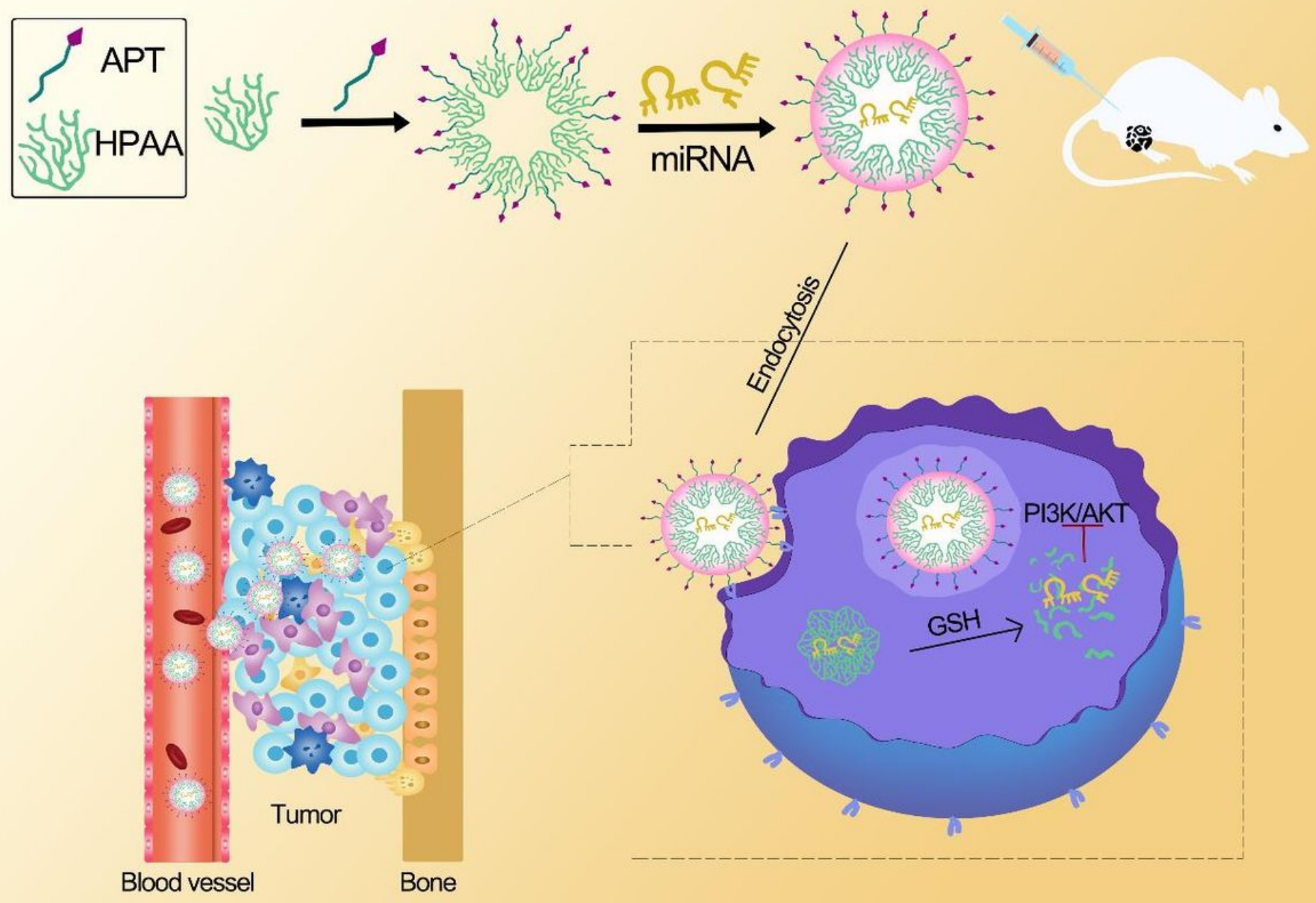

Figure 7

Scheme of the synthesis and the therapeutic effect to tumor of APT-PEG-HPAA/miRNA 